\title{
OSTEOTOMIA PROXIMAL DA TÍBIA: ESTABILIZAÇÃO DA ABERTURA MEDIAL COM ENXERTO TRICORTICAL DE ILÍACO
}

\author{
PROXIMAL TIBIAL OSTEOTOMY: STABILIZATION OF \\ THE MEDIAL OPENING WITH ATRICORTICAL ILIAC BONE GRAFT
}

\begin{abstract}
Roberto da Cunha Luciano', Getúlio Danival de Moura Souza ${ }^{2}$, Juliano Rispoli³ , Rodrigo Galvão Cardoso4,
\end{abstract} Marcus Vinícius Martins do Nascimento ${ }^{5}$, Gustavo Gontijo Domingos ${ }^{6}$, Dyego Vilela Luciano 7

\section{RESUMO}

Objetivo: Avaliação radiográfica do alinhamento do membro inferior, nos planos frontal e sagital, após osteotomia alta da tíbia. Para estabilização da osteotomia foi utilizado enxerto tricortical de ilíaco e parafuso de posicionamento. Métodos: Estudo prospectivo envolvendo 46 pacientes com idade entre 17 e 61 anos, sendo 42 portadores de geno varo secundário à osteoartrose e quatro por outras causas. A telerradiografia foi realizada para planejamento cirúrgico, utilizando o método de Frank Noyes modificado por Fugizawa. Foi realizado acesso cirúrgico convencional de $3 \mathrm{~cm}$ para retirada do enxerto tricortical de ilíaco. A osteotomia foi realizada sob controle de radioscopia, por incisão anteromedial de $3 \mathrm{~cm}$ com liberação da porção superficial do ligamento colateral medial. O enxerto foi colocado na porção posterior da osteotomia, para manter inalterado o slope tibial. O parafuso cruzou a osteotomia ortogonalmente para proteger a cortical lateral. Foram estabelecidos critérios radiográficos pré e pós-operatórios para avaliação dos resultados. Resultados: Houve consolidação em 100\% dos casos e manutenção do eixo mecânico, obtido no intraoperatório, em 94\%. A inclinação posterior do platô tibial, no plano sagital, variou entre $7^{\circ}$ e $12^{\circ}$. A mobilidade articular foi restabelecida em todos os pacientes operados. Onze pacientes apresentaram dor temporária no local da retirada do enxerto; no entanto, nenhum apresentou parestesia. A incidência de complicações foi de 8\% (infeç̧ão, perda da correção, fratura articular). Conclusão: A técnica mostrou-se reprodutível, simples, biológica, precisa e com baixos custos, podendo ser uma alternativa às técnicas já existentes.

\section{ABSTRACT}

Objective: Roentgenographic assessment of lower limb alignment, in the frontal and sagittal planes, after a high tibial osteotomy. To stabilize the osteotomy, a tricortical iliac graft was used along with a positioning screw. Methods: Prospective study of 46 patients with ages ranging from 17 to 61 years. Among them, 42 patients were carriers of genu varum secondary to knee osteoarthritis and four from other causes. Radiography was performed for surgical planning, using the Frank Noyes method modified by Fugizawa. Three $\mathrm{cm}$ conventional surgical access was performed to remove a tricortical iliac graft. The osteotomy was performed under fluoroscopic control, by $3 \mathrm{~cm}$ anteromedial incision with release of the superficial portion of the medial collateral ligament. The graft was placed in the posterior portion of the osteotomy to maintain an unaltered tibial slope. The screw crossed the osteotomy orthogonally to protect the lateral cortex. Radiographic criteria were established pre-and postoperatively to assess the results. Results: There was consolidation in $100 \%$ of cases and maintenance of the mechanical axis, obtained intraoperatively in $94 \%$ of cases. Correction of mechanical axis occurred in $94 \%$ (43 patients / 47 osteotomies). The posterior slope of the tibial plateau in the sagittal plane ranged from $7^{\circ}$ to $12^{\circ}$. Joint mobility was restored in all patients. Eleven patients had temporary pain at the site of graft removal, however, none had paraesthesia. The incidence of complications was $8 \%$ (infection, loss of correction, joint fracture). Conclusion: This technique is reproducible, simple, biologic, accurate, low-cost, and can be used as an alternative to existing techniques.

Descritores - Osteotomia; Joelho; Osteoartrite; Tíbia/cirurgia

Keywords - Osteotomy; Knee; Osteoarthritis; Tibia/surgery

1 - Chefe do Serviço de Cirurgia do Joelho do Hospital de Clínicas da Universidade Federal de Uberlândia, MG.

2 - Médico Estagiário do Serviço de Cirurgia do Joelho do Hospital de Clínicas da Universidade Federal de Uberlândia, MG.

3 - Ex-estagiário do Serviço de Ortopedia e Traumatologia da Universidade Federal de Uberlândia, MG.

4 - Médico Assistente do Departamento de Ortopedia e Traumatologia da Universidade Federal de Uberlândia, MG.

5 - Médico Residente do Serviço de Ortopedia e Traumatologia da Universidade Federal de Uberlândia, MG.

6 - Médico Assistente do Departamento de Ortopedia e Traumatologia da Universidade Federal de Uberlândia, MG.

7 - Aluno do Curso de Medicina da Universidade Federal de Uberlândia, MG.

Trabalho realizado no Hospital de Clínicas da Universidade Federal de Uberlândia, MG.

Correspondência: Avenida dos Vinhedos, 900 - Bairro Morada da Colina - CEP 38411-159 - Uberlândia, MG. E-mail: rluciano@orthomedcenter.com.br

Trabalho recebido para publicação: 05/10/09, aceito para publicação: 27/09/10. 


\section{INTRODUÇÃO}

As deformidades angulares do joelho têm causas variadas, entre elas a osteoartrose que é uma patologia inflamatória e degenerativa, com consequente caráter progressivo. Seu desfecho final é sempre a destruição do tecido condral e suas inerentes consequências ${ }^{(1,2)}$.

A osteotomia proximal da tíbia tem sido método de tratamento para as deformidades angulares do joelho há décadas. Foi pela primeira vez descrita por Jackson ${ }^{(3)}$ e posteriormente melhor estudada e difundida por Coventry et $a l^{(4)}$ e Insall et $a l^{(5)}$. O tratamento ortopédico através da osteotomia, corrigindo a deformidade angular, pode diminuir a velocidade de progressão deste fenômeno patológico.

A melhora das perspectivas prognósticas das condropatias degenerativas com o surgimento das terapias condroprotetoras e de viscossuplementação é uma realidade corroborada por estudos científicos ${ }^{(6)}$. Isto tem sido um dos motivos responsáveis pelo ressurgimento da osteotomia como mais uma possibilidade útil no arsenal terapêutico para a osteoartrose. Isto mesmo diante da melhora da qualidade e da sobrevida das próteses unicompartimentais ou totais. Quando indicada dentro dos critérios, já bem estabelecidos pela literatura ${ }^{(1,2)}$, a restauração do eixo mecânico do membro inferior e o consequente equilíbrio na distribuição de carga na articulação levam ao alívio da sintomatologia álgica e um inconteste ganho funcional com melhora da qualidade de vida do paciente ${ }^{(7,8)}$.

A osteotomia desvarizante proximal da tíbia é o procedimento preconizado para tratamento das deformidades angulares em varo do joelho, secundárias à osteoartrose do compartimento medial ou de outras etiologias. O procedimento classicamente descrito, e ainda muito utilizado, é a osteotomia em cunha de subtração lateral $^{(9)}$; no entanto, a osteotomia medial em cunha de adição tem apresentado vantagens, confirmadas pela literatura, que estimulam sua realização ${ }^{(10-12)}$.

Os resultados a médio e longo prazos dependem fundamentalmente da manutenção da correção, obtida no intraoperatório, após a consolidação da osteotomia. Para isto, o método de fixação é fundamental ${ }^{(13,14)}$. O enxerto tricortical de ilíaco mostrou-se suficientemente resistente para manter tal correção mesmo sem material de síntese adjuvante ${ }^{(15)}$. Permite, além da manutenção do alinhamento, a mobilização precoce, uma vez que o mecanismo de dobradiça formado pela cortical lateral e as estruturas miotendíneas e ligamentares laterais, as- sim como o aparelho extensor, garantem estabilidade suficiente para tal.

Na osteotomia em cunha de subtração determinadas complicações são relativamente frequentes. Entre elas podemos citar: retardo de consolidação, pseudartrose, recorrência da deformidade pela falta de estabilidade, impossibilidade de reposicionar o eixo mecânico após a realização dos cortes ósseos e as lesões neurovasculares potencializadas pela osteotomia da fíbula ${ }^{(16)}$. $\mathrm{Na}$ osteotomia em cunha de adição tais complicações praticamente inexistem. Outros fatores de relevância são a preservação do estoque ósseo do platô tibial e a não lateralização do mesmo em relação à diáfise da tíbia. Tais aspectos são fundamentais em uma eventual futura artroplastia total do joelho ${ }^{(11,12,15)}$.

O objetivo do presente estudo é avaliar os resultados radiográficos da técnica cirúrgica proposta e sua viabilidade. Analisamos o tempo de consolidação, a manutenção do eixo mecânico obtido com a correção cirúrgica e o ângulo de inclinação posterior do platô tibial (slope tibial) na radiografia em perfil verdadeiro.

\section{MÉTODOS}

O estudo, após análise da bibliografia, foi aprovado pela comissão de ética em pesquisa.

No período de agosto de 2004 a outubro de 2008 foram operados 46 pacientes (50 osteotomias), com idades variando entre 17 e 61 anos. Destes, três eram portadores de geno varo primário, sem artrose, e outro de deformidade em varo por vício de consolidação de fratura metafisária proximal da tíbia. Os demais pacientes (42) eram portadores de geno varo secundário a osteoartrose.

Seguiu-se o protocolo proposto para o estudo: a) avaliação clínica; b) avaliação radiográfica pré-operatória com radiografias AP e perfil com carga, axial de patela e telerradiografia dos membros inferiores; c) planejamento cirúrgico pela telerradiografia usando o método de Noyes ${ }^{(17)}$ (Figura 1); d) osteotomia em cunha de adição, mantendo o ângulo de abertura da mesma com enxerto tricortical de ilíaco e fixação com um parafuso de posição (Figura 2); e) imobilização com órtese inguinomaleolar por quatro semanas, porém com retirada da mesma a partir da segunda semana durante as sessões de fisioterapia; f) controles radiográficos semanais no primeiro mês e mensais até o final da $16^{\mathrm{a}}$ semana de pós-operatório; g) marcha com apoio parcial na sexta e total na $10^{a}$ semanas; h) telerradiografia dos membros inferiores no quinto mês pós-operatório. 


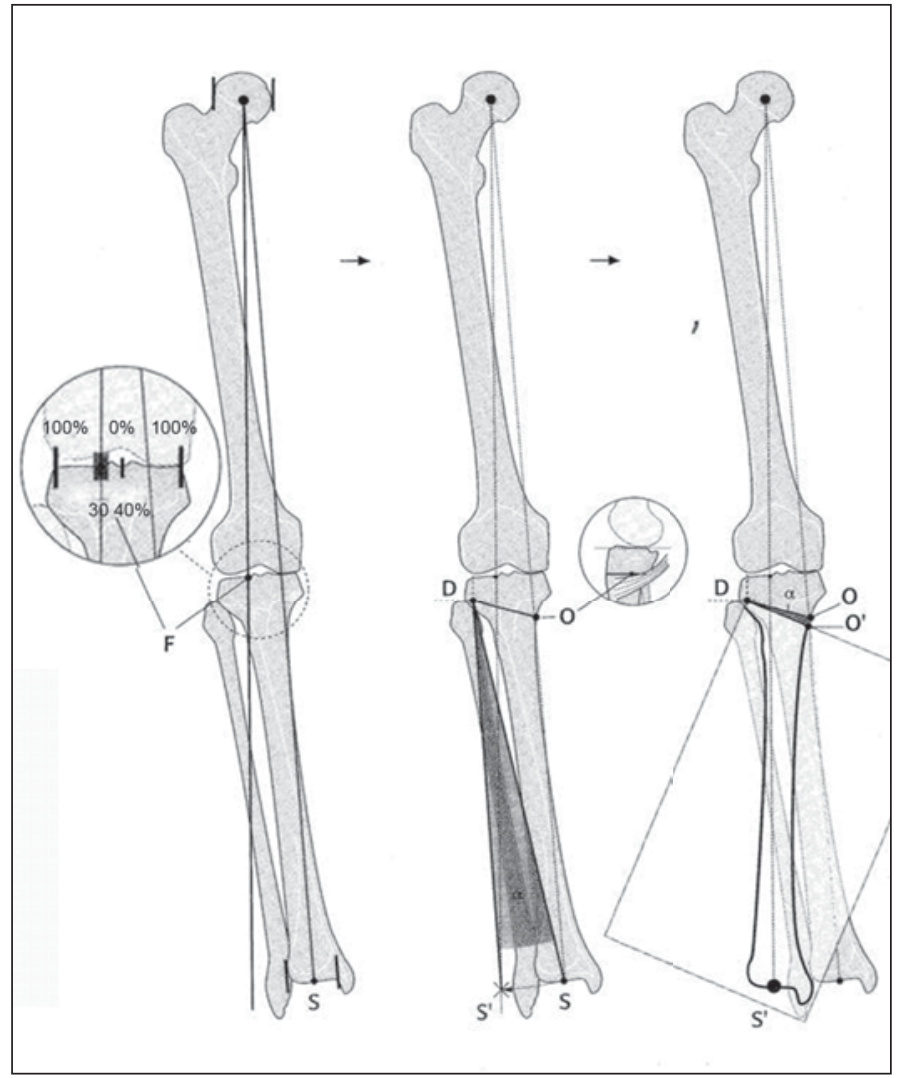

Figura 1 - Método de Noyes para planejamento da osteotomia a partir da telerradiografia.

Com o controle radiográfico (telerradiografia e radiografia em perfil da tíbia), calcula-se, respectivamente, o eixo mecânico do membro inferior corrigido e o ângulo de inclinação posterior (slope) do platô tibial.

A técnica cirúrgica consistiu na realização da retirada do enxerto tricortical de ilíaco, sendo este de comprimento aproximado de $3,0 \mathrm{~cm}$ e a base conforme os cálculos feitos no planejamento pré-operatório a partir da telerradiografia. Incisão anteromedial no joelho de $3,0 \mathrm{~cm}$, desinserção parcial e subperiostal dos tendões pata de ganso e subtotal da porção superficial do ligamento colateral medial (LCM), sob a inserção dos tendões flexores medias do joelho.

Osteotomia supratuberositária oblíqua, mantendo íntegra a cortical lateral na altura do ápice da cabeça da fíbula. Abertura da osteotomia e inserção do enxerto, até coincidir as corticais da base do enxerto com as da tíbia ao nível da osteotomia. Desta forma mantendo a abertura e consequentemente atingindo o alinhamento previamente calculado (eixo mecânico).

Um parafuso canulado (7.0), de posicionamento, passado de proximal-medial para distal-lateral, cruzando, em plano ortogonal, a osteotomia para proteger a cortical lateral (Figura 3).

Dreno de aspiração foi utilizado por 24 horas nas regiões de retirada do enxerto e na osteotomia. Antibioticoprofilaxia por 24 horas.

A técnica proposta para o estudo foi idealizada a partir de estudos já existentes e publicada na literatura ${ }^{(11,12,15)}$.
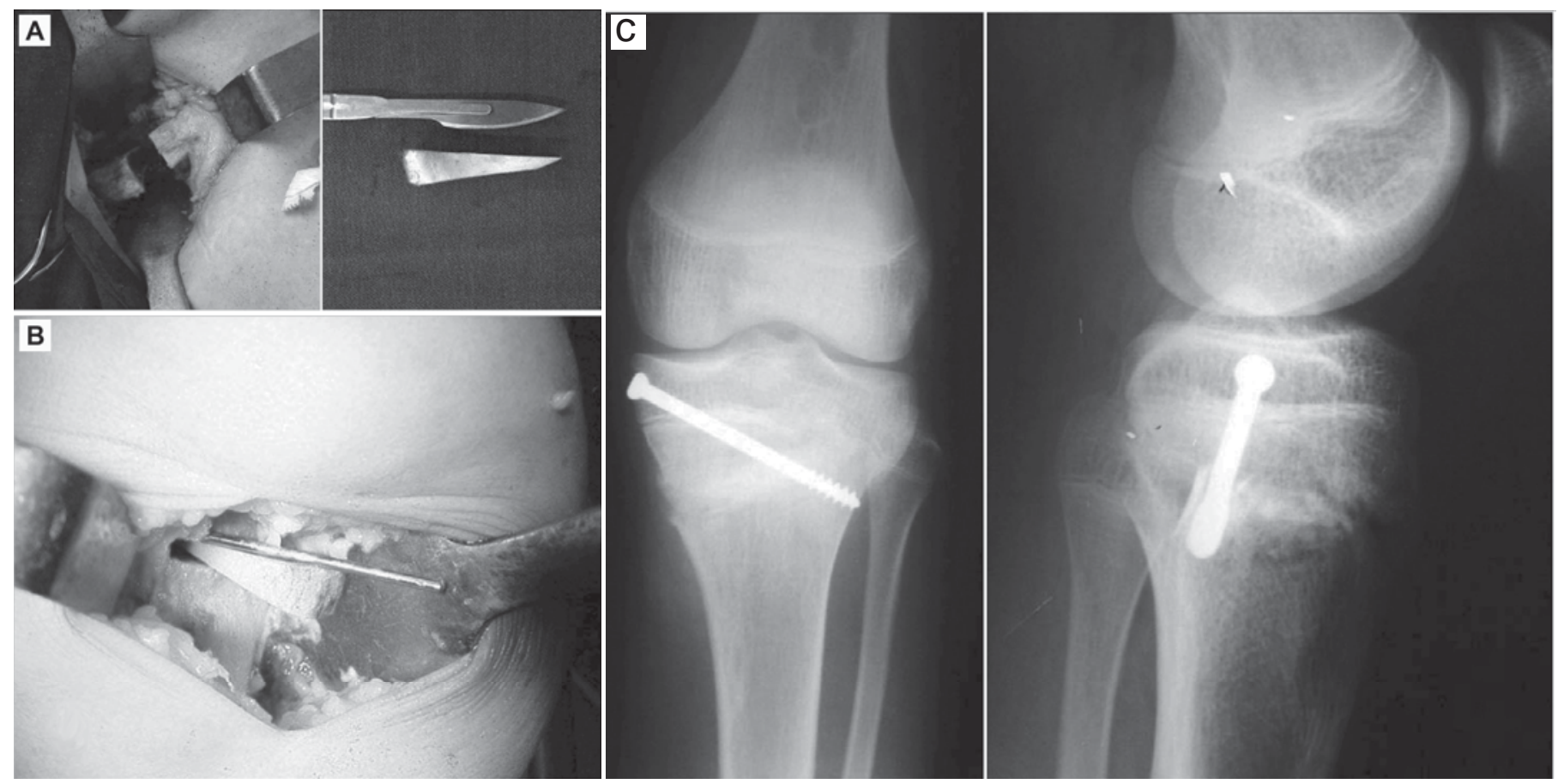

Figura 2 - Técnica cirúrgica: a) retirada de enxerto tricortical de ilíaco; b) manutenção do ângulo de abertura da osteotomia com enxerto; c) fixação com um parafuso de posição (radiografa pós-operatória). 

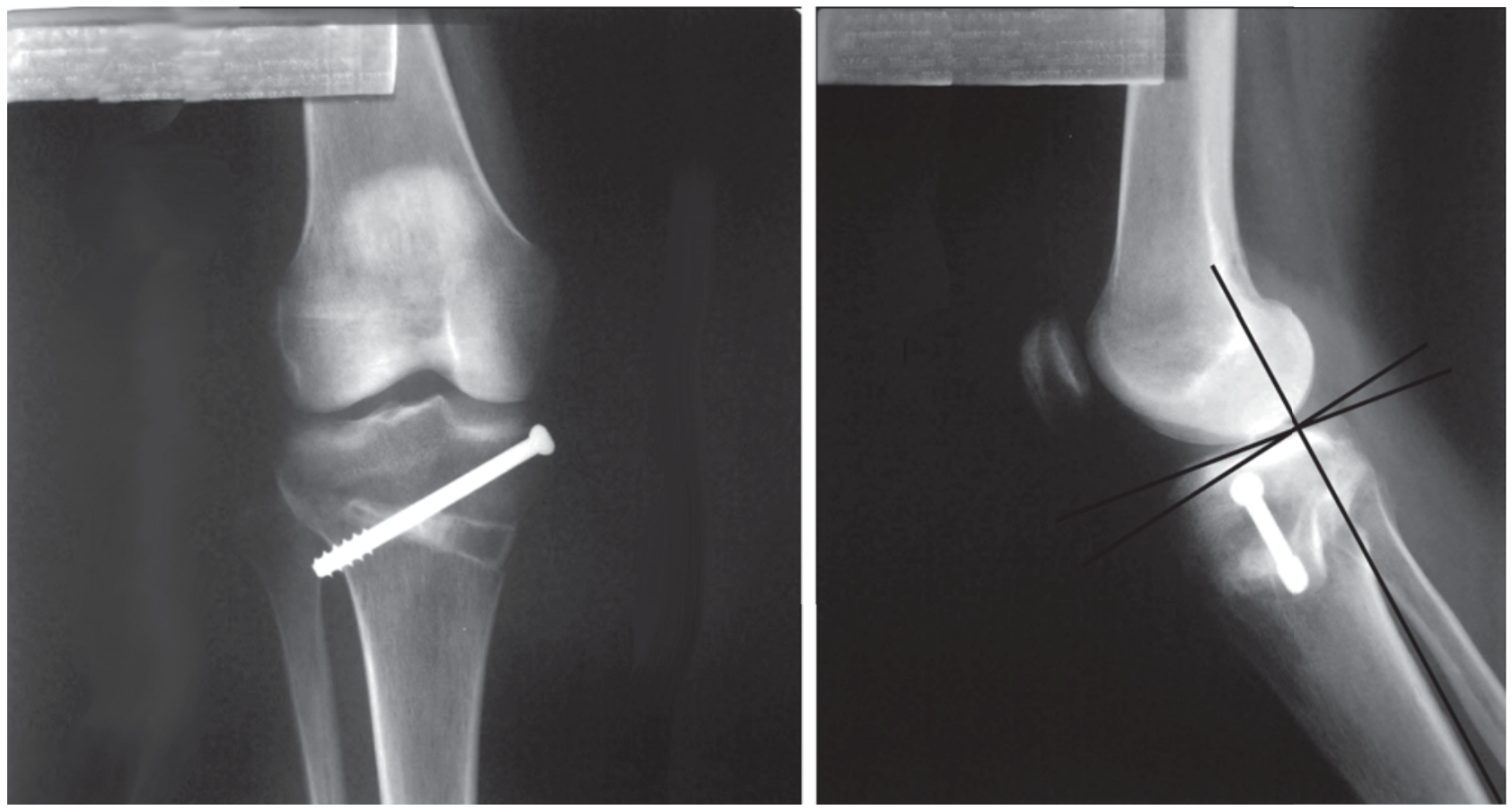

Figura 3 - Pós-operatório demonstrando parafuso de posição cruzando a osteotomia e o cálculo do ângulo de inclinação posterior do platô tibial (slope tibial).

\section{RESULTADOS}

A consolidação óssea ocorreu em um período máximo de 12 semanas em 100\% dos casos. Isto permitiu que a carga total fosse liberada neste período conforme o protocolo estabelecido.

O eixo mecânico, à avaliação radiográfica no plano coronal, obtido no intraoperatório, foi mantido em $94 \%$ (43 pacientes / 47 osteotomias). O ponto de passagem do eixo mecânico no platô tibial lateral, a partir do centro do joelho / ponto zero (Figura 4), apresentou a seguinte distribuição: $46 \%$ passou entre $40 \%$ e $35 \%$, 38\% pas-

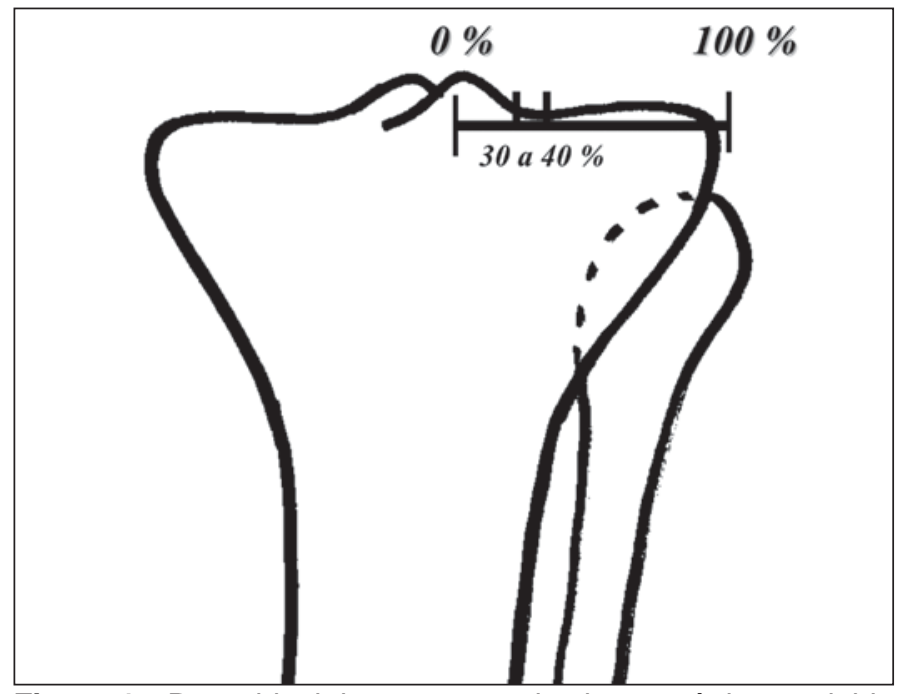

Figura 4 - Ponto ideal de passagem do eixo mecânico corrigido conforme preconizado por Noyes et al. sou entre $34 \%$ e $25 \%$, $8 \%$ passou entre 24 e $15 \%$, $6 \%$ passou entre $14 \%$ e $0 \%$ e $2 \%$ passou no planalto tibial medial (Tabela 1).

Tabela 1 - Ponto de passagem da linha de carga no platô tibial.

\begin{tabular}{c|c|c|c}
\hline Grupos & Passagem do eixo & $\begin{array}{c}\text { No de } \\
\text { osteotomias }\end{array}$ & $\begin{array}{c}\% \text { de } \\
\text { osteotomias }\end{array}$ \\
\hline A & Entre 35\% e 30\% & 23 & $46 \%$ \\
\hline B & Entre 29\% e 25\% & 19 & $38 \%$ \\
\hline C & Entre 24\% e 15\% & 4 & $8 \%$ \\
\hline D & Entre 14\% e 0\% & 3 & $2 \%$ \\
\hline E & Varo & 1 & \\
\hline
\end{tabular}

O ângulo de inclinação posterior da tíbia (slope tibial) variou entre $7^{\circ}$ e $12^{\circ}$. Foi feita a comparação entre a inclinação posterior (plano sagital) pré e pósoperatória (Tabela 2). Verificou-se não haver inversão

Tabela 2 - Inclinação sagital da superfície articular - slope tibial.

\begin{tabular}{c|c|c}
\hline $\begin{array}{c}\text { Inclinação posterior pós- } \\
\text { operatória. Plano sagital( }\end{array}$ & $\begin{array}{c}\text { № de } \\
\text { osteotomias }\end{array}$ & $\begin{array}{c}\% \text { de } \\
\text { osteotomias }\end{array}$ \\
\hline 0 & 12 & $24 \%$ \\
\hline 1 & 6 & $12 \%$ \\
\hline 2 & 17 & $34 \%$ \\
\hline 3 & 12 & $24 \%$ \\
\hline 4 & 3 & $6 \%$ \\
\hline
\end{tabular}

(*) Comparação com a inclinação (slope tibial) pré-operatória 
do slope tibial, mas em alguns casos houve aumento, mesmo colocando o enxerto mais posterior na abertura da osteotomia.

Não houve limitação da mobilidade articular ativa ou passiva em nenhum dos pacientes operados, inclusive nos reoperados.

Onze pacientes apresentaram dor no local de retirada do enxerto por um período entre seis e 10 semanas de pós-operatório.

A incidência de complicações foi de 8\% (quatro pacientes), sendo: infecção com perda do alinhamento em dois pacientes; um por subcorreção e um por fratura articular em consequência de osteotomia incompleta (Figura 5).

\section{DISCUSSÃO}

As mudanças mecânicas instituídas pela osteotomia de valgização no joelho varo, quando realizada sob rigorosos critérios de indicação, tem se traduzido em mudança da história natural da osteoartrite degenerativa ${ }^{(18)}$. Os relatos favoráveis apresentados pela literatura com relação às terapias condroprotetoras ${ }^{(6,19)}$, têm se tornado mais um motivo para se realizar procedimentos mais biológicos como as osteotomias. Por outro lado, não há na literatura consenso sobre os eventuais benefícios trazidos pela associação de procedimentos artroscópicos ${ }^{(16)}$. Em nosso estudo a artroscopia não foi realizada em nenhum dos pacientes.

A osteotomia em cunha de adição medial tem sido utilizada em número cada vez maior em função de algumas vantagens apresentadas, entre elas a da não necessidade de osteotomia da fíbula, a preservação do estoque ósseo da tíbia proximal, a previsibilidade na quantidade de graus a serem corrigidos, além de exigir menor dissecção cirúrgica. Isto, por si só, evita uma série de complicações como, por exemplo, a lesão do nervo fibular ${ }^{(20)}$. Não houve no nosso estudo nenhum caso de lesão do nervo fibular, ou de seus ramos.

A retirada de enxerto de ilíaco, quando realizada de acordo com as técnicas preconizadas pela literatura, é um procedimento de baixa morbidade, inclusive com baixa incidência de neuralgia ilioinguinal ${ }^{(21)}$. Na nossa casuística houve persistência da dor no sítio de retirada do enxerto em oito pacientes, por um período máximo de 10 semanas. Dados estes coincidentes com os apresentados pela literatura ${ }^{(19)}$.

A hipercorreção, através das osteotomias valgizantes, com passagem do eixo mecânico pelo planalto tibial lateral é fundamental para a obtenção de bons resultados ${ }^{(1,2)}$. Com a técnica descrita no estudo, obtivemos correção considerada ideal, passando entre $40 \%$ e $30 \%$ do platô tibial lateral, em $84 \%$ dos casos. Obteve-se ainda $8 \%$ em que o ponto de passagem da linha de carga ficou entre $29 \%$ e $25 \%$. A inclinação do platô tibial no plano sagital é um ponto crítico a ser tecnicamente observado. A colocação do enxerto posteriormente na osteotomia é necessário para a manutenção do slope tibial dentro de parâmetros mecanicamente aceitáveis ${ }^{(22)}$. Na nossa casuística pôde-se observar uma variação entre $7^{\circ}$ e $12^{\circ}$, não havendo, no entanto a inversão do slope tibial.

\section{CONCLUSÃO}

A técnica cirúrgica desenvolvida no presente estudo mostrou-se reprodutível, manteve o aspecto biológico pela mínima quantidade de implante utilizado. Além de apresentar custos inferiores à maioria das técnicas existentes para 0 mesmo fim, obtendo as mesmas taxas de consolidação.

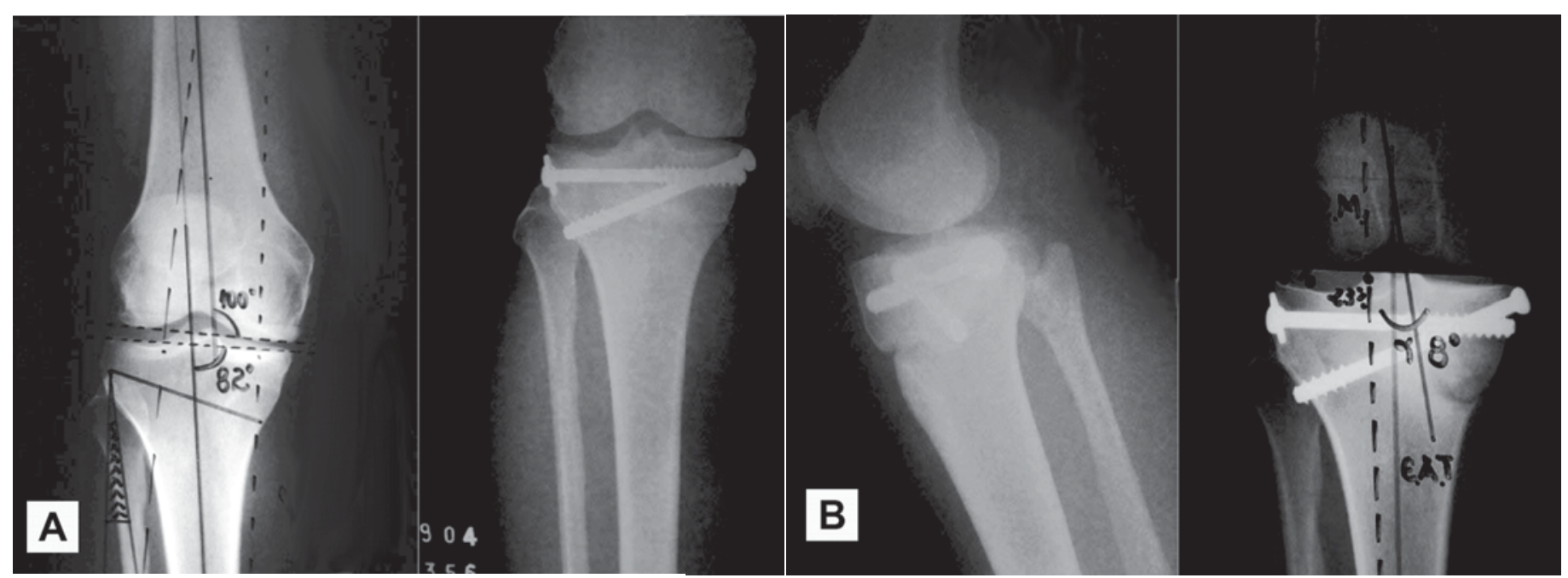

Figura 5 - Caso descrito como complicação, com fratura articular devido à osteotomia incompleta, fixada no intraoperatório. 


\section{REFERÊNCIAS}

1. Camanho GL. Tratamento da osteoartrose do joelho. Rev Bras Ortop. 2001;36(5):135-40.

2. Camanho GL, Olivi R, Camanho LF. Técnica de fixação para osteotomia supratuberositária cupuliforme valgizante da tíbia. Rev Bras Ortop. 2001;36(7):263-7.

3. Jackson JP. Osteotomy for osteoarthrites of the knee. J Bone Joint Surg Br. 1958;40:826.

4. Coventry MB, Ilstrup DM, Wallrichs SL. Proximal tibial osteotomy: A critical longterm study of eighty seven cases. J Bone Joint Surg Am. 1993;75(2):196-201.

5. Insall JN, Joseph DM, Msika C. High tibial osteotomy for varus gonarthrosis: A long-term follow-up study. J Bone Joint Surg Am. 1984;66(7):1040-8.

6. Brief AA, Maurer SG, Di Cesare. Use of glicosamine and chondroitina sulfate in the management of osteoarthritis. J Am Acad Orthop Surg. 2001;9(2):71-8.

7. Molina DR, Sado JJ, Mendlovitz PSR, Rubin MLL, Camanho GL. Avaliação da deformidade rotacional do joelho em pacientes com genuvaro e osteoartrose. Rev Bras Ortop. 2002;37(10):430-5.

8. Keyes GW, Carr AJ, Miller RK, Goodfellow WJ. The arthrographic classification of medial gonarthrosis. Correlation with operation methods in 200 knees. Acta Orthop Scand. 1992;63(5):497-501.

9. Magyar G, Ahl TL, Vibe P, Toksvig-Larsen S, Lindstrand A. Open-wedge osteotomy by hemicallotasis or the closed-wedge technique for osteoarthritis of the knee. A randomised study of 50 operations. J Bone Joint Surg Br. 1999;81(3):444-8.

10. Nakamura E, Mizuta H, Kudo S, Takagi K, Sakamoto K. Open-wedge osteotomy of the proximal tíbia with hemicallotasis. J Bone Joint Surg $\mathrm{Br}$. 2001;83(8):1111-5

11. Staubli AE, Simoni C, Babst R, Lobenhoffer P. TomoFix: a new LCP-concept for open wedge osteotomy of the medial proximal tibia - early results in 92 cases. Injury. 2003;34(Suppl 2):B55-62.
12. Hernigou P, Ma W. Open wedge tibial osteotomy with acrylic bone cement as bone substitute. Knee. 2001;8(2):103-10.

13. Billings A, Scott DF, Camargo MP, Hofmann AA. High tibial osteotomy with a calibrated osteotomy guide, rigid inter al fixation, and early motion: long-term follow-up. J Bone Joint Surg Am. 2000;82(1):70-9.

14. Hofmann AA, Wyatt RW, Beck SW. High tibial osteotomy. Use of an osteotomy jig, rigid fixation, and early motion versus conventional surgical technique and cast immobilization. Clin Orthop Relat Res. 1991;(271):212-7.

15. Patond KR, Lokhande AV. Medial open wedge high tibial osteotomy in medial compartment osteoarthrosis of the knee. Natl Med J India. 1993;6(3):104-8.

16. Wright JM, Crockett HC, Slawski DP, Madsen MW, Windsor RE. High tibial osteotomy. J Am Acad Orthop Surg. 2005;13(4):279-98

17. Dugdale TW, Noyes FR, Styer D. Preoperative planning for high tibial osteotomy. The effect of lateral tibiofemoral separation and tibiofemoral length. Clin Ortop Relat Res. 1992;(274):248-64.

18. Kanamiya T, Naito M, Yoshimura I: The influence of biomechanical factorson cartilage regeneration after high tibial osteotomy for knees with medial compartiment osteoarthrites: Clinical and arthroscopic observations. Arthroscopy. 2002;18(7):725-9.

19. Raman R, Dutta A, Day N, Sharma HK, Shaw CJ, Johnson GV. Efficacy of Hylan G-F 20 and Sodium Hyaluronate in the treatment of osteoarthritis of the knee - a prospective randomized clinical trial. Knee. 2008;15(4):318-24.

20. Kirgs A, Albrecht S. Palsy of the deep peroneal nerve after proximal tibial osteotomy. An anatomical study. J Bone Joint Surg Am. 1992;74(8):1180-5.

21. Smith S E, DeLee JC, Ramamurthy S. Ilioinguinal neuralgia following iliac bone-grafting. Report of two cases and review of the literature. J Bone Joint Surg Am. 1984;66(8):1306-8.

22. Noyes FR, Goebel SX, West J. Opening wedge tibial osteotomy: the 3-triangle method to correct axial alignment and tibial slope. Am J Sports Med. 2005;33(3):378-87. 